\title{
Dynamics of spin transport in voltage-biased Josephson junctions
}

\author{
Erhai Zhao and J. A. Sauls \\ Department of Physics \& Astronomy, Northwestern University, Evanston, IL 60208
}

(Dated: April 18, 2018)

\begin{abstract}
We investigate spin transport in voltage-biased spin-active Josephson junctions. The interplay of spin filtering, spin mixing, and multiple Andreev reflection leads to nonlinear voltage dependence of the $d c$ and $a c$ spin current. We compute the voltage characteristics of the spin current $\left(I_{\mathrm{S}}\right)$ for superconductor-ferromagnet-superconductor (SFS) Josephson junctions. The sub-harmonic gap structure of $I_{\mathrm{S}}(V)$ is shown to be sensitive to the degree of spin mixing generated by the ferromagnetic interface, and exhibits a pronounced even-odd effect associated with spin transport during multiple Andreev reflection processes. For strong spin mixing both the magnitude and the direction of the $d c$ spin current can be sensitively controlled by the bias voltage.
\end{abstract}

PACS numbers: 72.25.-b, 72.25.Mk, 74.50.+r

By controlling the quasiparticle spin degree of freedom in solid-state heterostructures, spintronics provides a new paradigm and possibilities for making devices and circuits with improved functionality over conventional electronics [1]. Although spintronics research has been mostly focused on hybrid structures of normal metal $(\mathrm{N})$, semiconductors, and ferromagnetic materials, superconductors has increasingly proved to be a useful component in constructing spintronic devices. This is because the proximity coupling of superconductor $(\mathrm{S})$ to ferromagnet $(\mathrm{F})$, on the scale ranging from nanometers to microns, offers a unique opportunity to probe the spin degree of freedom and coherently control the spin polarized current [2, 3]. For example, two leading methods of measuring the spin polarization of ferromagnetic materials, spin-dependent tunnelling [4] and point contact Andreev reflection spectroscopy [5], both rely on measuring the conductance of superconductor-ferromagnet junctions [1, 6]. Recently spin-triplet supercurrent was created to flow through the superconductor-ferromagnetsuperconductor (SFS) Josephson junctions [7]. The spin current transported through in SFNFS structures was also predicted to have properties with the potential of making superconducting spin-transfer devices [8, 9$]$.

The equilibrium properties of SF hybrid systems have been extensively studied in recent years 2, 3]. However, nonequilibrium spin-transport properties, which are essential for the purpose of manipulating spin current flow, are far from being well understood. As an example, the SFS $\pi$ junction has been proposed for constructing an environmentally decoupled quantum bit [10]. Optimizing its performance requires knowledge of its dissipative dynamics under nonequilibrium conditions, such as the current-voltage characteristics which have only been studied in certain limiting cases [16, 27].

In this paper we present a new, general theoretical framework to compute the time-dependent transport properties of SF hybrids. Compared to previous approaches
[1, 12, 13], our method allows one to find analytically the time-dependent nonequilibrium (Keldysh) Green's functions near spin-active interfaces. We apply the formalism to compute the spin current-voltage characteristics of voltage-biased SFS junctions to show that SFS structures can serve as highly tunable spin filters as well as spin-current oscillators for spintronic applications.

We consider SFS point contacts to illustrate the dynamical properties of spin-active Josephson junctions. The left (1) and right (2) leads are assumed to be spinsinglet, $s$-wave superconductors $(\mathrm{S})$ in the clean limit. The ferromagnetic metallic layer $(\mathrm{F})$ is assumed to have a homogeneous magnetization fixed in the $\hat{\mathbf{z}}$ direction by anisotropy energy. We also assume the $\mathrm{F}$ layer is specular and that its thickness is much less than the superconducting coherence length and the mean free path in the leads. The contact is characterized by an S-matrix 19]. Each S-matrix component is a $2 \times 2$ spin matrix, which for a fixed quantization axis for spin, $\hat{\mathbf{z}}$, is diagonal: $S_{11}=S_{22}=\operatorname{diag}\left(\sqrt{R_{\uparrow}} e^{i \vartheta / 2}, \sqrt{R_{\downarrow}} e^{-i \vartheta / 2}\right), S_{21}=S_{12}=$ $\operatorname{diag}\left(i \sqrt{D_{\uparrow}} e^{i \vartheta / 2}, i \sqrt{D_{\downarrow}} e^{-i \vartheta / 2}\right)$. $D_{\alpha}$ is the transmission probability for normal state conduction electrons with spin projection $\alpha=\uparrow, \downarrow$, and $R_{\alpha}=1-D_{\alpha}$. In general $D_{\uparrow} \neq D_{\downarrow}$, so the F layer acts like a "spin filter"; $\vartheta$, referred to as the "spin mixing angle", is the relative phase between the transmitted (and reflected) spin up and down electrons. Spin mixing at the F layer leads to rotation of the direction of the spin polarization analogous to Faraday rotation of linearly polarized photons [14]. Particle-hole symmetry relates the S-matrix for quasiholes to that for quasi-particles, $\underline{S}_{i j}\left(\mathbf{p}_{f_{\| \mid}}\right)=S_{j i}\left(-\mathbf{p}_{f_{\|}}\right)^{\operatorname{tr}}$ [20], which simplifies for junctions with inversion symmetry to $\underline{S}_{i j}=S_{i j} . D_{\alpha}$ and $\vartheta$ can be calculated from material parameters such as the exchange field, the Fermi wave vector mismatch (FWM), and the F layer thickness; see section III of Ref. 19 for detailed discussions on the interface S-matrix. We emphasize that FWM plays an important role in FS junctions. For example, as shown in [6], the subgap conductance of a FS junction is very sen- 
sitive to FWM and a nonmonotonic function of spin polarization. For transition metal ferromagnets such as Co and $\mathrm{Ni}$, the transmission probabilities, $D_{\alpha}$, range from 0.2 to 0.9 , while $\vartheta$ can take any value between 0 and $\pi$, depending on the F layer thickness [21].

For NFN junctions with normal metallic leads (N) under voltage bias $V$, the spin current flowing through the junction is due to spin filtering and linear in $V, I_{\mathrm{s}}=G_{\mathrm{s}} V$. The spin conductance $G_{\boldsymbol{S}} \propto\left(D_{\uparrow}-D_{\downarrow}\right)$ is independent of the spin-mixing angle. Spin mixing, on the other hand, plays a central role in transport through SFS junctions. For example, it can give rise to $\pi$-junction behavior [2, 15], as well as complex features in the IV characteristics of the charge current, i.e. the "sub-harmonic gap structure" (SGS) [16]. For voltage-biased SFS contacts, the effective transmission probability for spin up (down) quasiparticles in the superconducting leads depends not only on $D_{\uparrow}$ and $D_{\downarrow}$, but also on the spin-mixing angle $(\vartheta)$ and the time-dependent Josephson phase difference across the contact, $\phi(t)=2 \mathrm{eVt} / \hbar$. The latter leads to inelastic scattering of quasiparticles of energy $\epsilon$ by the spin-active interface, i.e. multiple Andreev reflection (MAR) [17, 18], which transfers quasiparticles inelastically into side-band states with energies $\epsilon_{n} \equiv \epsilon+n \hbar \omega$, with $\hbar \omega \equiv e V$. As a result a time-dependent spin current flows across the junction which has $d c$ and $a c$ components with frequencies $2 n \omega$,

$$
\mathbf{I}_{\mathrm{S}}(t)=\mathbf{I}_{0}+\sum_{n=1}^{\infty}\left(\mathbf{I}_{n}^{c} \cos 2 n \omega t+\mathbf{I}_{n}^{s} \sin 2 n \omega t\right) .
$$

Our goal is to find the dependence of $\left\{\mathbf{I}_{0}, \mathbf{I}_{n}^{c / s}\right\}$ on the bias voltage and scattering parameters $\left\{D_{\alpha}, \vartheta\right\}$.

Our method for computing time-dependent transport properties is based on formulating the quasiclassical equations of nonequilibrium superconductivity 22] in terms of particle-hole coherence functions and distributions functions, collectively known as the Riccati amplitudes. These functions obey Riccati-type transport equations and uniquely determine the quasiclassical Green's functions. The Riccati formulation is discussed in Refs. [19, 23]; we follow the notation of these authors. For superconductors in the clean limit the coherence function $\gamma^{R}\left(\tilde{\gamma}^{R}\right)$ is the local probability amplitude for a hole (electron) being converted into an electron (hole). The distribution functions $x^{K}$ and $\tilde{x}^{K}$ describe the nonequilibrium occupation of the particle and hole states. Quasiparticle scattering near the contact, including spin filtering, spin mixing and MAR, is described by a set of time-dependent boundary conditions for the Riccati amplitudes. We have derived these boundary conditions for spin-active interfaces in Ref. [19]. Here we present exact solutions for the boundary conditions for the case of a constant bias voltage. The local Green's functions at the contact are constructed from the Riccati amplitudes and the spin

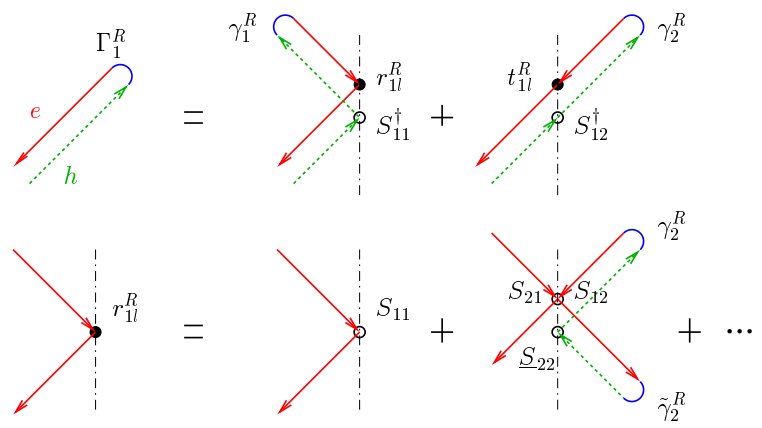

FIG. 1: Diagrammatic representation of Eqs. (2). The solid (dash) arrows represent electrons (holes), the vertical line indicates the interface. Branch conversion amplitudes $\left(\gamma^{R}\right.$ and $\left.\tilde{\gamma}^{R}\right)$ are shown as semi-circles connecting electron and hole lines, interface scattering amplitudes $\left(S_{i j}\right)$ are shown as circles $(\circ)$, and the effective reflection and transmission amplitudes $\left(r_{1 l}^{R}\right.$ and $\left.t_{1 l}^{R}\right)$ are indicated by bullets $(\bullet)$. Only the leading order multiple scattering processes for $r_{1 l}^{R}$ are shown.

current is calculated from the Keldysh Green's function, $\hat{\mathfrak{g}}^{K}$. We have checked that our method reproduces known results for nonequilibrium charge transport through spinindependent Josephson junctions [17, 18, 24, 25], as well as the SFS structure studied in Ref. [16].

We set the phase $\phi_{1}=0$ and $\phi_{2}=\phi(t)$ and work in a gauge where $\phi(t)=2 \mathrm{eVt} / \hbar$. The corresponding Riccati amplitudes for the two leads are (in the energy domain): $\gamma_{1}^{R}\left(\epsilon^{\prime}, \epsilon\right)=2 \pi \delta\left(\epsilon^{\prime}-\epsilon\right) \gamma_{1}^{R}(\epsilon), x_{1}^{K}\left(\epsilon^{\prime}, \epsilon\right)=2 \pi \delta\left(\epsilon^{\prime}-\epsilon\right) x_{1}^{K}(\epsilon)$, $\gamma_{2}^{R}\left(\epsilon^{\prime}, \epsilon\right)=2 \pi \delta\left(\epsilon^{\prime}-\epsilon+2 \omega\right) \gamma_{2}^{R}(\epsilon-\omega)$, and $x_{2}^{K}\left(\epsilon^{\prime}, \epsilon\right)=$ $2 \pi \delta\left(\epsilon^{\prime}-\epsilon\right) x_{2}^{K}(\epsilon+\omega)$. The remaining Riccati amplitudes are obtained from those above by symmetry relations: $\gamma_{i}^{A}=\left[\tilde{\gamma}_{i}^{R}\right]^{\dagger}$ and $\tilde{q}\left(\hat{\mathbf{p}}_{f}, \epsilon^{\prime}, \epsilon\right) \equiv q^{*}\left(-\hat{\mathbf{p}}_{f},-\epsilon^{\prime},-\epsilon\right)[23]$. Note that $\gamma_{2}^{R}$ and $\tilde{\gamma}_{2}^{R}$ act as ladder operators in energy space.

Consider a set of scattering trajectories $\{1 i, 1 o, 2 i, 2 o\}$ near the contact (" $i "=$ incoming and " $o "=$ outgoing), c.f. Fig. 1 of Ref. [19]. Boundary conditions relate "outgoing" and "incoming" Riccati amplitudes via the interface S-matrix. For example, $\Gamma_{1}^{R}$ on trajectory $1 i$ is given by,

$$
\begin{aligned}
\Gamma_{1}^{R} & =r_{1 l}^{R} \circ \gamma_{1}^{R} \underline{S}_{11}^{\dagger}+t_{1 l}^{R} \circ \gamma_{2}^{R} \underline{S}_{12}^{\dagger}, \\
r_{1 l}^{R} & =\left[S_{11}^{\dagger}-\beta_{21} \circ \beta_{22}^{-1} S_{12}^{\dagger}\right]^{-1}, t_{1 l}^{R}=-r_{1 l}^{R} \circ \beta_{21} \circ \beta_{22}^{-1}
\end{aligned}
$$

where $\beta_{i j} \equiv S_{i j}^{\dagger}-\gamma_{j}^{R} \underline{S}_{i j}^{\dagger} \circ \tilde{\gamma}_{i}^{R}$, and the convolution is defined as $[x \circ y]\left(\epsilon^{\prime}, \epsilon\right)=\int d \epsilon^{\prime \prime} x\left(\epsilon^{\prime}, \epsilon^{\prime \prime}\right) y\left(\epsilon^{\prime \prime}, \epsilon\right) / 2 \pi$ in energy domain. Equations (2) are the summation of multiple scattering amplitudes represented by the diagrams shown in Fig. [1 Constructing $\hat{\mathfrak{g}}^{K}$ from the Ricatti amplitudes then leads us to evaluate the product,

$$
\mathrm{A} \equiv\left(1-\Gamma_{1}^{R} \circ \tilde{\gamma}_{1}^{R}\right)^{-1} \circ r_{1 l}^{R}=\left[\beta_{11}-\beta_{21} \circ \beta_{22}^{-1} \circ \beta_{12}\right]^{-1} .
$$

The ladder properties of $\gamma_{2}^{R}$ and $\tilde{\gamma}_{2}^{R}$ lead to $\mathrm{A}\left(\epsilon^{\prime}, \epsilon\right)=$ $\sum_{m} 2 \pi \delta\left(\epsilon^{\prime}-\epsilon-m \omega\right) \mathrm{A}_{m}(\epsilon)$, where $\mathrm{A}_{m}(\epsilon)$ has the phys- 
ical interpretation of the transition amplitude for qusiparticles of energy $\epsilon$ in lead 1 to be scattered into energy $\epsilon_{m}$. The calculation of $\left\{\mathrm{A}_{m}\right\}$ requires inverting an infinite dimensional tri-diagonal matrix in energy space, or equivalently solving the three-term recurrence equation,

$$
o_{m} \mathrm{~A}_{m}+p_{m} \mathrm{~A}_{m-2}+q_{m} \mathrm{~A}_{m+2}=\delta_{m 0},
$$

where the coefficients are defined by

$$
\begin{aligned}
o_{m}= & \beta_{11}\left(\epsilon_{m}\right)-S_{21}^{\dagger} \beta_{22}^{-1}\left(\epsilon_{m+1}\right) S_{12}^{\dagger} \\
& -p_{m}(\epsilon)\left[S_{12}^{\dagger}\right]^{-1} \gamma_{2}^{R}\left(\epsilon_{m-1}\right) \underline{S}_{12}^{\dagger} \tilde{\gamma}_{1}^{R}\left(\epsilon_{m}\right), \\
p_{m}= & \gamma_{1}^{R}\left(\epsilon_{m}\right) \underline{S}_{21}^{\dagger} \tilde{\gamma}_{2}^{R}\left(\epsilon_{m-1}\right) \beta_{22}^{-1}\left(\epsilon_{m-1}\right) S_{12}^{\dagger}, \\
q_{m}= & S_{21}^{\dagger} \beta_{22}^{-1}\left(\epsilon_{m+1}\right) \gamma_{2}^{R}\left(\epsilon_{m+1}\right) \underline{S}_{12}^{\dagger} \tilde{\gamma}_{1}^{R}\left(\epsilon_{m+2}\right) .
\end{aligned}
$$

Equation (4) is solved by introducing transfer matrices, $Z_{m}^{ \pm}$, such that $\mathrm{A}_{m}=Z_{m}^{+} \mathrm{A}_{m-2}$ for $m>0$ and $\mathrm{A}_{m}=$ $Z_{m}^{-} \mathrm{A}_{m+2}$ for $m<0$. The $Z_{m}^{ \pm}$can be expressed as matrix continued fractions $Z_{m}^{+}=\left[o_{m}+q_{m} Z_{m+2}^{+}\right]^{-1}\left(-p_{m}\right), Z_{m}^{-}=$ $\left[o_{m}+p_{m} Z_{m-2}^{-}\right]^{-1}\left(-q_{m}\right)$, and evaluated using standard procedures [26]. The resulting $Z_{m}^{ \pm}$allow us to compute $\mathrm{A}_{m}$ for even $m$ starting from $\mathrm{A}_{0}=\left[o_{0}+p_{0} Z_{-2}^{-}+q_{0} Z_{2}^{+}\right]^{-1}$. The amplitudes for odd $m$ vanish identically.

The angle- and energy-resolved spin-current evaluated to the left of the contact is given by $\mathbf{J}_{s}^{l}\left(\hat{\mathbf{p}}_{f}\right)=$ $g\left(\hat{\mathbf{p}}_{f}\right) \operatorname{Tr}\left[\boldsymbol{\sigma} \hat{\tau}_{3}\left(\hat{\mathfrak{g}}_{1 i}^{K}-\hat{\mathfrak{g}}_{1 o}^{K}\right)\right] / 2 \pi i$, where the trace is over particle-hole and spin space, $g\left(\hat{\mathbf{p}}_{f}\right)=N_{f} \mathcal{A} \frac{\hbar}{2}\left(\mathbf{v}_{f} \cdot \hat{\mathbf{n}}\right), N_{f}$ is the density of states at the Fermi level, $\mathbf{v}_{f}$ is the Fermi velocity, $\mathcal{A}$ is the contact area, and $\hat{\mathbf{n}}$ is the normal to the junction interface. We evaluate the current by expressing $\hat{\tau}_{3}\left(\hat{\mathfrak{g}}_{1 i}^{K}-\hat{\mathfrak{g}}_{1 o}^{K}\right)$ in terms of the local Riccati amplitudes and carry out the trace over particle-hole space. We obtain a sum of 8 terms; e.g. the contributions from electronlike quasiparticles from lead 1 , which are proportional to $x_{1}^{K}$, are $\mathrm{A} \circ x_{1}^{K} \circ \mathrm{A}^{\dagger}$ and $\tilde{\gamma}_{1}^{R} \circ \mathrm{A} \circ x_{1}^{K} \circ \mathrm{A}^{\dagger} \circ \gamma_{1}^{A}$. Similarly, contributions from $\tilde{x}_{1}^{K}, x_{2}^{K}$, and $\tilde{x}_{2}^{K}$ are expressed as convolutions with transition amplitudes B, C, D, and E, which can all be expressed in terms of A [see Eq. (9)]. We evaluate the convolution in the energy domain, then Fourier transform to obtain the trajectory-resolved dynamical spin current,

$$
\mathbf{J}_{s}^{l}\left(\hat{\mathbf{p}}_{f} ; t\right)=g\left(\mathbf{p}_{f}\right) \operatorname{Re} \sum_{k, m} e^{i 2 k \omega t} \int \frac{d \epsilon}{2 \pi} \operatorname{Sp}\left[\boldsymbol{\sigma} \mathrm{K}_{m k}(\epsilon)\right],
$$

where $\mathrm{Sp}$ is a trace over spin space. The kernel $\mathrm{K}_{m k}(\epsilon)$ consists of 8 terms which describe interference between inelastic scattering $\epsilon \rightarrow \epsilon_{2 m}$ and $\epsilon \rightarrow \epsilon_{2(m+k)}$ :

$$
\begin{aligned}
\mathrm{K}_{m k}(\epsilon) & =\mathrm{H}_{m k}(\epsilon)+\tilde{\gamma}_{1}^{R}\left(\epsilon_{2 m}\right) \mathrm{H}_{m k}(\epsilon)\left[\tilde{\gamma}_{1}^{R}\left(\epsilon_{2(m+k)}\right)\right]^{\dagger} \\
& -\mathrm{D}_{2 m} \tilde{x}_{1}^{K}(\epsilon) \mathrm{D}_{2(m+k)}^{\dagger}-\mathrm{E}_{2 m} \tilde{x}_{1}^{K}(\epsilon) \mathrm{E}_{2(m+k)}^{\dagger}, \\
\mathrm{H}_{m k}(\epsilon) & =\mathrm{A}_{2 m} x_{1}^{K}(\epsilon) \mathrm{A}_{2(m+k)}^{\dagger}+\mathrm{B}_{2 m} x_{2}^{K}\left(\epsilon_{1}\right) \mathrm{B}_{2(m+k)}^{\dagger} \\
& -\mathrm{C}_{2 m} \tilde{x}_{2}^{K}(\epsilon-1) \mathrm{C}_{2(m+k)}^{\dagger}, \\
\mathrm{B}_{m}(\epsilon) & =\left[\mathrm{A}_{m-2}\left(\epsilon_{2}\right) \gamma_{1}^{R}\left(\epsilon_{2}\right) \underline{S}_{21}^{\dagger} \tilde{\gamma}_{2}^{R}\left(\epsilon_{1}\right)-\mathrm{A}_{m}(\epsilon) S_{21}^{\dagger}\right] \beta_{22}^{-1}\left(\epsilon_{1}\right),
\end{aligned}
$$
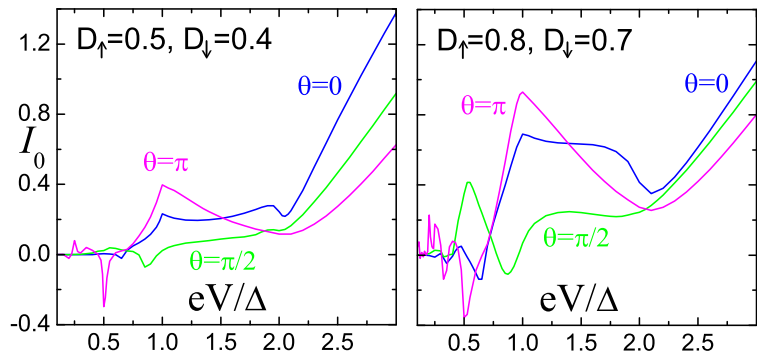

FIG. 2: The $d c$ spin current (in units of $G_{\mathrm{s}} \Delta / e$ ) as a function of voltage bias $V$ at zero temperature, for different transmission probabilities, $D_{\uparrow}$ and $D_{\downarrow}$, and spin-mixing angles, $\vartheta=0$ (blue), $\pi / 2$ (green), $\pi$ (red).

$$
\begin{aligned}
\mathrm{C}_{m}(\epsilon) & =\left\{\mathrm{D}_{m}(\epsilon) \underline{S}_{12}-\left[\mathrm{A}_{m+2}\left(\epsilon_{-2}\right) S_{11}^{\dagger}\right.\right. \\
& \left.\left.+\mathrm{B}_{m+2}\left(\epsilon_{-2}\right) S_{12}^{\dagger}\right] S_{12} \gamma_{2}^{R}\left(\epsilon_{-1}\right)\right\} \tilde{\beta}_{22}^{-1}\left(\epsilon_{-1}\right), \\
\mathrm{D}_{m}(\epsilon) & =\mathrm{A}_{m}(\epsilon) \gamma_{1}^{R}(\epsilon) \underline{S}_{11}^{\dagger}+\mathrm{B}_{m+2}\left(\epsilon_{-2}\right) \gamma_{2}^{R}\left(\epsilon_{-1}\right) \underline{S}_{12}^{\dagger}, \\
\mathrm{E}_{m}(\epsilon) & =\tilde{\gamma}_{1}\left(\epsilon_{m}\right)\left[\mathrm{A}_{m}(\epsilon) S_{11}^{\dagger}+\mathrm{B}_{m}(\epsilon) S_{12}^{\dagger}\right] \tilde{\gamma}_{1}^{-1}(\epsilon) .
\end{aligned}
$$

Thus, the calculation of the spin current is reduced to the calculation of the $\left\{\mathrm{A}_{m}\right\}$. Integration over all contributing trajectories yields the total spin current on the left side of the junction, $\mathbf{I}_{s}^{l}(t)=\left\langle\mathbf{J}_{s}^{l}\left(\hat{\mathbf{p}}_{f}, t\right)\right\rangle$, where $\langle\ldots\rangle=\int_{0}^{\pi / 2} d \psi \sin (2 \psi)(\ldots) / 4$, and $\psi=\arccos \left(\hat{\mathbf{v}}_{f} \cdot \hat{\mathbf{n}}\right)$. Eqs. (8)-(9) are our central results. They are valid for very general contact scattering matrices.

In general spin-active junctions do not conserve the spin current. However, for the SFS point contacts described above, only the $\mathbf{z}$ component of spin current is nonzero and it is conserved across the contact, $\mathbf{I}_{s}(t)=I_{s}(t) \hat{\mathbf{z}}$. Furthermore, the suppression of order parameter near the point contact, as well as spin accumulation in the leads, are negligible. Thus, $\gamma_{j}^{R}(\epsilon), \tilde{\gamma}_{j}^{R}(\epsilon), x_{j}^{K}(\epsilon)$, and $\tilde{x}_{j}^{K}(\epsilon)$ can be evaluated in terms of their bulk values in the corresponding leads [19]. For the results reported below we neglected the directional dependence of $D_{\alpha}$ and $\vartheta$ and assumed an inelastic scattering rate of $10^{-4} \Delta$. The IV characteristics, when normalized by the temperaturedependent gap $\Delta(T)$, depend only weakly on the temperature, so we only present results for $T=0$.

Figure 2 shows the $d c$ component of spin current at zero temperature for two sets of spin dependent transparencies, and three values of the spin mixing angle. Consider first the case of pure spin filtering, i.e. $\vartheta=0$. For $e V>2 \Delta$, the spin current is always less than the normal state value, $G_{\mathrm{S}} V$, where the spin conductance $G_{\mathrm{S}}=N_{f} v_{f} \frac{\hbar}{2} \mathcal{A}\left\langle D_{\uparrow}-D_{\downarrow}\right\rangle$. The SGS of the spin current shows anomalies at voltages, $V_{n}=2 \Delta / n e$, which are more pronounced in higher transmission junctions. The structures associated with even and odd harmonics are different: the spin current is a local minimum near $V_{2 m+1}$, but a local maximum near $V_{2 m}$. The SGS for the spin current is in sharp contrast with that of the 


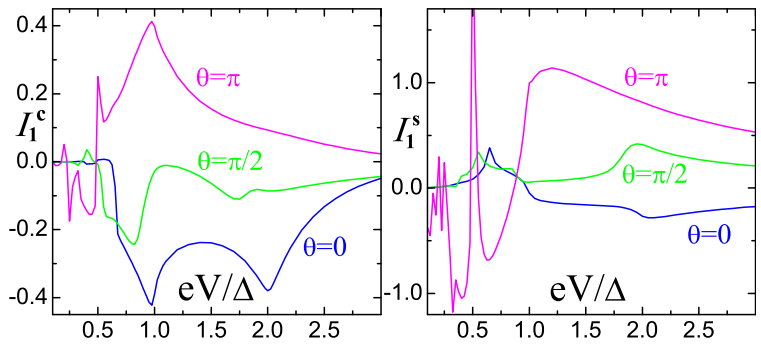

FIG. 3: Left (right): The cosine (sine) components of the $a c$ spin current (in units of $G_{\mathrm{s}} \Delta / e$ ) at $T=0$ for $\vartheta=0, \pi / 2, \pi$, $D_{\uparrow}=0.5$, and $D_{\downarrow}=0.4$.

charge current [16]. The deficit in spin current at high voltages, as well as the even-odd effect in the SGS reflect the quasiparticle origin of the spin current. Multiple Andreev reflection processes starting at subgap energies contribute significantly to the charge current, but much less so to the spin current. Consider a scattering event where an incoming spin-up electron-like quasiparticle of energy $\epsilon \lesssim-\Delta$ in lead 1 climbs up the MAR ladder to energy $\epsilon_{n} \gtrsim \Delta$ after $n-1$ Andreev reflections $(n \sim 2 \Delta / e V)$ and leaks into the leads. This process transfers charge ne to lead 2 , but only spin angular momentum $\hbar / 2$, and only if $n$ is odd. The $n^{\text {th }}$ order MAR process has an onset voltage, $V_{n}$. Opening up new transmission channels at $V_{2 m+1}$ leads to increase of the spin current, while the onset of back scattering at $V_{2 m}$ leads to decrease in spin current. For larger contact transparencies contributions from higher-order MAR processes become relevant, and more detailed structures in the IV curve develop at low voltages, as are evident in the right panel of Fig. 2

The inclusion of spin mixing leads to interface bound states at energies $\Delta \cos (\vartheta / 2)$ [spin up] and $-\Delta \cos (\vartheta / 2)$ [spin down] [15]. Qualitatively, this structure of the local density of states (LDOS) affects the spin transport in two ways. First, the LDOS for continuum states near the gap edge is depleted. This leads to suppression of the spin current at high voltages; also the SGS at the onset voltages for MAR processes is less pronounced. Second, scattering events with MAR trajectories which coincide with the bound states exhibit resonant transmission and dominate the spin current. Thus, in relatively lowtransmission junctions the SGS is displaced from $V_{n}$ to voltages near $\Delta(1+\cos (\vartheta / 2)) / n$ [16]. Since the resonances for spin up and spin down quasiparticles occur at different voltages, the $d c$ spin current oscillates with voltage, even reversing direction in junctions with moderate to high transparency. For $\vartheta=\pi$, the bound states are degenerate and located at the Fermi level, and consequently the SGS at $V_{n}$ is recovered.

Figure 3 shows the cosine and sine part of the first Fourier component of the $a c$ spin current at zero temperature for intermediate transparencies: $D_{\uparrow}=0.5, D_{\downarrow}=0.4$. Note that the magnitudes of $I_{1}^{c}$ and $I_{1}^{s}$ are comparable to that of the $d c$ current. However, higher order Fourier components of the $a c$ spin current are generally smaller in magnitude, but readily calculated from Eqs. (8)-(9) . As in the case of the $d c$ current, the structures in $I_{1}^{c}(V)$ and $I_{1}^{S}(V)$ are related to onsets and resonances associated with MAR processes. Resonant transmission leads to dramatic variations and sharp peaks in the $a c$ spin current at voltages less than $2 \Delta$; e.g. for $\vartheta=\pi$ the sinecomponent dominates the response near $\mathrm{eV} / \Delta=0.5$ and reverses sign over a narrow voltage range, $e \delta V / \Delta \approx 0.1$.

In conclusion, we calculated the dynamical spin current in voltage-biased spin-active Josephson contacts. The dependence of the spin current on the voltage bias exhibits nonlinearities and resonant structures that are in striking contrast to the IV characteristics of the charge current, but which can be understood in terms of spin-filtering, spin-mixing and MAR. The nonlinear spin IV characteristics may be used to control the magnitude and the direction of the spin current via bias voltage, and may be of interest for superconducting spintronic devices.

We thank Tomas Löfwander for discussions on ac Josephson effects.

[1] I. Zutic, J. Fabian, and S. D. Sarma, Rev. Mod. Phys. 76, 323 (2004).

[2] A. I. Buzdin, Rev. Mod. Phys. 77, 935 (2005).

[3] F. S. Bergeret et al, Rev. Mod. Phys. 77, 1321 (2005).

[4] P. M. Tedrow and R. Meservey, Phys. Rep. 238, 173 (1994).

[5] R. J. Soulen et al, Science 282, 85 (1998).

[6] I. Zutic and O. T. Valls, Phys. Rev. B 60, 6320 (1999).

[7] R. S. Keizer et al, Nature 439, 825 (2006).

[8] X. Waintal and P. W. Brouwer, Phys. Rev. B 65, 054407 (2002).

[9] C. Bell et al, Appl. Phys. Lett. 84, 1153 (2004).

[10] L. B. Ioff et al, Nature 398, 679 (1999).

[11] I. C. Cuevas and M. Fogelström, Phys. Rev. B 64, 104502 (2001).

[12] D. Huertas-Hernando, Yu. V. Nazarov, and W. Belzig, Phys. Rev. Lett. 88, 047003 (2002).

[13] J. Kopu et al, Phys. Rev. B 69, 094501 (2004).

[14] T. A. Tokuyasu, J. A. Sauls, and D. Rainer, Phys. Rev. B38, 8823 (1988).

[15] M. Fogelström, Phys. Rev. B 62, 11812 (2000).

[16] M. Andersson, J. C. Cuevas, and M. Fogelström, Physica C 367, 117 (2002).

[17] T. M. Klapwijk, G. E. Blonder, and M. Tinkham, Physica B+C 109-110, 1657 (1982).

[18] E. N. Bratus, V. S. Shumeiko, and G. Wendin, Phys. Rev. Lett. 74, 2110 (1995).

[19] E. Zhao, T. Löfwander, and J. A. Sauls, Phys. Rev. B 70, 134510 (2004).

[20] A. Millis, D. Rainer, and J. A. Sauls, Phys. Rev. B38, 
4504 (1988).

[21] M. Stiles, J. Appl. Phys. 79, 5805 (1996).

[22] G. M. Eliashberg, Sov. Phys. JETP 34, 668 (1972).

[23] M. Eschrig, Phys. Rev. B 61, 9061 (2000).

[24] D. V. Averin and A. Bardas, Phys. Rev. Lett. 75, 1831 (1995).

[25] J. C. Cuevas, A. Martin-Rodero, and A. L. Yeyati, Phys.
Rev. B 54, 7366 (1996).

[26] H. Risken and H. D. Vollmer, Z. Physik B 39, 339 (1980).

[27] I. V. Bobkova, Phys. Rev. B 73, 012506 (2006). This paper studies spin current in SFS tunnel junctions. Applying our exact formula to the tunneling limit yields different result from the approximate solution in Ref. 27; these differences will be addressed elsewhere. 